\title{
Estudos bioquímios e histopatológicos do fígado de ratos wistar alimentados com Spirulina cepa LEB-18 em tamanho micrométrico e nanométrico
}

\author{
Biochemical and histopathological studies of the liver of wistar rats fed Spirulina strain LEB-18 in \\ micrometric and nanometric size
}

Estudios biológicos e histopatológicos del hígado de ratas wistar alimentadas con Spirulina cepa LEB-18 en tamaño micrométrico y nanométrico

Recebido: 03/01/2021 | Revisado: 04/01/2021 | Aceito: 21/01/2021 | Publicado: 25/01/2021

\begin{tabular}{|c|c|}
\hline Instituto Federal de Educaç & $\begin{array}{r}\text { Adriana Rodrigues Machado } \\
\text { ORCID: https://orcid.org/0000-0002-2641-256X } \\
\text { Universidade Federal do Rio Grande, Brasil } \\
\text { E-mail: adririzo85@ @mail.com } \\
\text { Rosane da Silva Rodrigues } \\
\text { ORCID: https://orcid.org/0000-0001-8172-2053 } \\
\text { Universidade Federal de Pelotas, Brasil } \\
\text { E-mail: rosane.rodrigues @ ufpel.edu.br } \\
\text { Leticia Marques de Assis } \\
\text { ORCID: https://orcid.org /0000-0003-2477-2112 } \\
\text { ção, Ciência e Tecnologia Sul-rio-grandense, Brasil } \\
\text { E-mail: leticiamassis@ gmail.com } \\
\text { Carmen Lucia Garcez Ribeiro } \\
\text { ORCID: https://orcid.org/0000-0002-2500-0563 } \\
\text { Universidade Federal de Pelotas, Brasil } \\
\text { E-mail: caluribeiro@ yahoo.com.br } \\
\text { Andrea da Silva Ramos Rocha } \\
\text { ORCID: https://orcid.org/0000-0003-2234-0033 } \\
\text { Universidade Federal de Pelotas, Brasil } \\
\text { E-mail: andrearochavet@ gmail.com } \\
\text { Mirian Ribeiro Galvão Machado } \\
\text { ORCID: https://orcid.org/0000-0001-7505-7659 } \\
\text { Universidade Federal de Pelotas, Brasil } \\
\text { E-mail: miriangalvao@gmail.com } \\
\text { Leonor Almeida de Souza-Soares } \\
\text { ORCID: https://orcid.org/0000-0002-8688-4761 } \\
\text { Universidade Federal do Rio Grande, Brasil } \\
\text { E-mail: Leonor.souzasoares@ gmail.com }\end{array}$ \\
\hline
\end{tabular}

\begin{abstract}
Resumo
O objetivo do estudo foi avaliar as respostas bioquímicas e histopatológicas de ratos fêmeas alimentados por 15 dias com dietas contendo Spirulina cepa LEB 18 no tamanho de micrômetro (Sm) e nanômetro (Sn), dieta aproteica (A) e dieta controle (C) contendo caseína. Foram utilizadas 24 fêmeas recém-desmamadas (21 dias) da linhagem Wistar / UFPEL. As análises realizadas foram: lipídios nas dietas, excrementos e fígado, cálculo da digestibilidade lipídica, determinações bioquímicas séricas do colesterol total e frações (LDL, HDL e VLDL) e triacilgliceróis e glicemia, e avaliação histopatológica dos fígados. As dietas contendo Spirulina LEB 18 nos tamanhos micro e nanométricos não alteraram parâmetros bioquímicos (colesterol total e frações, triacilgliceróis e glicose) de ratos Wistar em crescimento. Dados fisiológicos e bioquímicos de ratos submetidos à dieta (A) indicam comprometimento do metabolismo lipídico, devido à ausência de proteína, o que reflete diretamente em lesões hepáticas bastante heterogêneas quanto ao padrão e intensidade em relação (aos) às demais dietas, e pode ser devido à deposição lipídica mais evidenciada em uma dieta aproteica, provavelmente causada por deficiência de proteínas e, portanto, pelo declínio na produção e exportação de lipoproteínas pelo fígado.
\end{abstract}

Palavras-chave: Fígado; Rattus norvegicus; Spirulina.

\section{Abstract}

The aim of the study was to evaluate the biochemical and histopathological responses of rats fed for 15 days on diets containing Spirulina strain LEB 18 in micrometer size $(\mathrm{Sm})$ and nanometer (Sn), an aproteic diet (A) and a control diet (C) containing casein. 24 recently weaned (21 days) female rats of the Wistar/UFPEL strain were used. The analyses carried out were: lipids in the diets, (excreta) feces and liver, and calculation of lipid digestibility, serum biochemical determinations of total cholesterol and fractions (LDL, HDL and VLDL) and triacylglycerols and blood 
glucose, and histopathological evaluation of the livers. The diets containing Spirulina LEB 18 in the micro and nanometer sizes did not alter biochemical parameters (total and fractions cholesterol, triacylglycerols and glucose) of Wistar rats in growth. Physiological and biochemical data from rats subjected to diet (A) indicate an impairment in the metabolism of lipids, due to the absence of protein in it, which directly reflected in hepatic lesions that were very heterogeneous regarding the pattern and intensity in relation to the other diets, and may be due to lipid deposition more evidenced in an aproteic diet, probably caused by protein deficiency and therefore the decline in production and export of lipoproteins by the liver.

Keywords: Liver; Rattus norvegicus; Spirulina.

\section{Resumen}

El objetivo del estudio fue evaluar las respuestas bioquímicas e histopatológicas de las ratas alimentadas durante 15 días con dietas que contenían la cepa de Spirulina cepa LEB - 18 en tamaño de micrómetro (Sm) y nanómetro (Sn), una dieta aproteica (A) y una dieta de control (C ) que (contiene) contenia caseína. Se utilizaron 24 ratas hembras destetadas recientemente (21 días) de la cepa Wistar / UFPEL. Los análisis realizados fueron: lípidos en las dietas, excretas (e) y hígado, y cálculo de la digestibilidad de los lípidos, determinaciones bioquímicas séricas de colesterol total y fracciones (LDL, HDL y VLDL) y triacilgliceroles y glucosa en sangre, y evaluación histopatológica de los hígados. Las dietas que contenían Spirulina cepa LEB-18 en los tamaños micro y nanométricos no alteraron los parámetros bioquímicos (colesterol total y fracciones, triacilgliceroles y glucosa) de ratas Wistar en crecimiento. Los datos fisiológicos y bioquímicos de las ratas sometidas a la dieta (A) indican un deterioro en el metabolismo de los lípidos, debido a la ausencia de proteínas, que se refleja directamente en las lesiones hepáticas que eran muy heterogéneas con respecto al patrón y la intensidad en relación con el otro. dietas, y puede deberse a la deposición de lípidos más evidenciada en una dieta aproteica, probablemente causada por deficiencia de proteínas y, por lo tanto, la disminución en la producción y exportación de lipoproteínas por el hígado.

Palabras clave: Hígado; Rattus norvegicus; Spirulina.

\section{Introdução}

A Spirulina platensis tem sido extensivamente estudada e destaca-se por sua qualidade nutricional, índice proteico de alto valor biológico, aminoácidos essenciais, além de outros componentes como vitaminas, minerais e ácidos graxos essenciais, principalmente ácido gama-linolênico e $\beta$-caroteno (Belay, 1997, Machado et al., 2017). Além destes compostos, suas células apresentam produtos do metabolismo secundário, que induzem efeitos fisiológicos, como, por exemplo, os compostos fenólicos (ácido cafeico, clorogênico, salicílico, trans-cinâmico), tocoferol e pigmentos (carotenoides, ficocianina e clorofila), aos quais são atribuídas suas propriedades potencialmente funcionais (Assis, 2012; Machado et al., 2015). Ao mesmo tempo, apresenta algumas vantagens sobre outras algas, como aumento da palatabilidade, sem problemas na digestão nem toxicidade aparente para os seres humanos, o que não ocorre com algas como Chlorella e Scenedesmus (Contreras et al.1979).

A microalga Spirulina possui atividade antioxidante e propriedade hipocolesterolêmica, é classificada como GRAS (geralmente reconhecido como seguro) pelo FDA (Food and Drug Administration), que garante seu uso como alimento sem riscos à saúde. No Brasil, a Spirulina tem sido utilizada principalmente na produção de cápsulas para dietas de emagrecimento, embora muitos estudos mostrem outros usos, como controle do metabolismo do colesterol e de suas frações (RodriguezHernandez et al., 2001).

De acordo com Cushen (2012), a nanotecnologia tem sido uma ajuda potencial aos avanços na produção de alimentos de qualidade com propriedades funcionais aprimoradas. Os prováveis benefícios do uso dessas nanopartículas em alimentos estão relacionados à facilidade de alcançar órgãos devido ao aumento da absorção, auxiliando os efeitos nutricionais e funcionais em comparação com o tamanho macrométrico do material. Assim, estima-se que a ingestão de Spirulina em tamanho nanométrico possa trazer mais benefícios para o organismo pelo consumo desta microalga.

O objetivo deste estudo foi avaliar as alterações bioquímicas e histopatológicas no fígado de ratos Wistar / UFPel alimentados com dieta contendo Spirulina cepa LEB -18 nos tamanhos de micrômetro e nanômetro. 


\section{Material e Métodos}

A presente pesquisa caracterizou-se como um estudo laboratorial (quantitativo) e qualitativo, com isso os métodos qualitativos são aqueles nos quais é importante a interpretação por parte do pesquisador com suas opiniões sobre o fenômeno em estudo, assim como a torna-se importante a prática reflexiva de ênfase social que se investiga e do processo de investigação (Pereira, et al. 2018).

\subsection{Material}

A biomassa da microalga Spirulina cepa LEB-18 foi fornecida pelo Laboratório de Engenharia Bioquímica (LEB), isolada da lagoa Mangueira (33 $302133 \mathrm{~S}, 53^{\circ} 082593$ W), localizada em Santa Vitória do Palmar, RS, Brasil (Morais et al, 2008) e suplementada com $20 \%$ do meio Zarrouk (Costa, Colla, Duarte- Fllho, 2004). Os ingredientes para a preparação de dietas foram comprados no comércio ou em empresas especializadas. No entanto, a mistura mineral (Reeves et al., 1993) foi obtida por pesagem e homogeneização dos minerais p.a., enquanto a mistura vitamínica foi adquirida em farmácia de manipulação.

\subsection{Métodos}

\subsubsection{Preparação de Spirulina de tamanho nanométrico}

A Spirulina cepa LEB-18 foi fornecida seca na forma de péletes e posteriormente foi triturada em moinho de bolas (Modelo Q298-2) e peneirada em agitador de peneiras, de malha 200 mesh, para homogeneização do tamanho de partícula, atingindo um tamanho de partícula de $88 \mu \mathrm{m}$. Após foi acondicionada a vácuo em embalagens de polietileno de alta densidade (PEAD), com capacidade para $500 \mathrm{~g}$, e armazenada sob refrigeração à temperatura de $\pm 7^{\circ} \mathrm{C}$, até o momento da análise.

A Spirulina cepa LEB-18 com diâmetro de 88 micrômetros foi dissolvida em tampão fosfato de sódio pH 7,0, 0,2 M, com subsequente aquecimento em banho-maria, com vórtice e homogeneização em um Ultra-Turrax a 10.000 rpm por 20

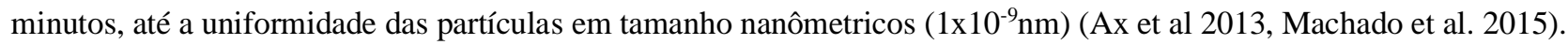

\subsubsection{Preparação das dietas}

As dietas foram formuladas, como mostrado na Tabela 1, para atender às necessidades básicas de um rato durante o crescimento (Reeves et al., 1993). Foram preparadas quatro dietas, sendo uma de controle (C) contendo caseína, uma aproteica (A) e duas dietas contendo Spirulina cepa LEB-18: uma com a microalga em tamanho micormétrico (Sm) e a outra em tamanho manométrico (Sn). As dietas seguiram as recomendações do AIN-93 (American Institute of Nutrition) para roedores (Reeves et al., 1997). 
Tabela 1. Dietas experimentais elaboradas e administradas durante 15 dias a ratos fêmeas da cepa Wistar/UFPel $\left(\mathrm{g} \mathrm{Kg}^{-1}\right)$.

\begin{tabular}{|c|c|c|c|c|}
\hline \multirow[b]{2}{*}{ *Ingredientes } & \multicolumn{4}{|c|}{$* *$ Dietas } \\
\hline & $\mathbf{C}$ & $\mathbf{A}$ & Sm & Sn \\
\hline Spirulina cepa LEB-18 & - & - & 200 & - \\
\hline Spirulina Nanometrica & - & - & - & 80 \\
\hline Caseina & 160 & - & - & 80 \\
\hline Óleo de soja & 70 & 70 & 70 & 70 \\
\hline Mistura mineral & 35 & 35 & 35 & 35 \\
\hline Mistura vitamínica & 10 & 10 & 10 & 10 \\
\hline L- cistina & 3.0 & 3.0 & 3.0 & 3.0 \\
\hline Bitartarato de Colina & 2.5 & 2.5 & 2.5 & 2.5 \\
\hline Sacarose & 100 & 100 & 100 & 100 \\
\hline Farelo de trigo & 50 & 50 & 50 & 50 \\
\hline Amido de milho & 569.5 & 729.5 & 529.5 & 569.5 \\
\hline
\end{tabular}

* Fontes de nutrientes: caseína comercial (SIGMA), L-cistina, bitartarato de colina, mistura mineral, mistura de vitaminas (farmácia de manipulação), óleo de soja, sacarose, farelo de trigo e amido de milho comprados em lojas locais. ${ }^{*}$ Dieta: $\mathrm{C}=\mathrm{Controle;} \mathrm{A}=\mathrm{Aproteica} ; \mathrm{Sm}$ = Spirulina micrometrica; $\mathrm{Sn}=$ Spirulina nanometrica

Fonte: Reeves et al. (1993), adaptado.

\subsubsection{Ensaio biológico}

O bioensaio foi realizado no Laboratório de Experimentação Animal do Centro de Ciências Químicas, Farmacêuticas e de Alimentos, da Universidade Federal de Pelotas (UFPel), com aprovação do Comitê de Ética da Universidade Federal de Pelotas e Experimentação Animal (CEEA) sob o número de registro 23110.000978 / 2010-91. Foram utilizadas 24 ratas fêmeas recém-desmamadas (21 dias) (Rattus norvegicus), da (linhagem) cepa Wistar / UFPEL do viveiro da Universidade Federal de Pelotas, pesadas e colocadas aleatoriamente em gaiolas metabólicas individuais, onde receberam a dieta controle (C) por 4 dias como período de adaptação às condições ambientais.

Após o período de adaptação, os animais foram pesados e redistribuídos em quatro grupos $(n=6)$ por sorteio, obtendo-se o peso médio dos grupos de $56 \mathrm{~g}$, aproximadamente. As dietas isocalóricas foram fornecidas em $20 \mathrm{~g} / \mathrm{dia}$ e água ad libitum, durante 15 dias. Os animais foram mantidos em gaiolas metabólicas individuais, em ambiente com temperatura constante $\left(24^{\circ} \mathrm{C} \pm 2^{\circ} \mathrm{C}\right)$, troca de ar por exaustão a cada $12 \mathrm{~h}$ e iluminação adequada (ciclo claro e escuro de 12 horas). Durante o período experimental de 15 dias, os restos das dietas foram pesados todos os dias, e os ratos semanalmente, para verificar o consumo e o ganho de peso. Nesse período, também foi realizada a coleta de fezes para posterior determinação dos lipídios pelo método de Bligh e Dyer (1959) adaptado por Araújo (2009). No final do experimento, os animais foram mantidos em jejum por 12 horas e eutanasiados por decapitação.

\subsubsection{Remoção do fígado e avaliação bioquímica}

Após a eutanásia, alíquotas de sangue foram coletadas e imediatamente depositadas em fitas especiais para medição de glicose e lidas no equipamento Accu-Chek Advantage II (Roche $\left.{ }^{\circledR}\right)$. O sangue restante foi coletado e centrifugado a $1000 x$ $g$ por 15 minutos a $4{ }^{\circ} \mathrm{C}$, separando o soro que foi congelado $\left(-20^{\circ} \mathrm{C}\right)$ para a determinação de triacilglicerois e colesterol total e VLDL, HDL, LDL e minerais em Equipamento Biosystems BTS -310" semi-automático com uso de reagentes e instrumentos reativos da Biosystems ${ }^{\circledR}$.

Após a abertura do abdômen, os fígados foram coletados, lavados em solução salina fria ( $\mathrm{NaCl}$ a $0,9 \%)$, secos em papel filtro, pesados, sendo um lóbulo destinado às avaliações histopatológicas e o restante embrulhado em papel alumínio e 
congelado a $-20{ }^{\circ} \mathrm{C}$ para determinações subsequentes de lipídios. As carcaças, bem como os demais materiais descartáveis utilizados, foram embaladas separadamente em sacos apropriados e enviados, através do Biotério, para descarte especializado.

\subsubsection{Determinação de lipídios nas fezes e digestibilidade lipídica}

Para determinação de lipídios, primeiramente as fezes foram coletadas e armazenadas sob refrigeração, e após, secas em estufa a $50 \pm 1{ }^{\circ} \mathrm{C}$ por 48 horas. Foram moídas (almofariz e pistilo) alíquotas de $3 \mathrm{~g}$, provenientes de cada, animal. As determinações foram realizadas, em triplicata, de acordo com Bligh e Dyer (1959) adaptado por Araújo (2009). Os resultados foram calculados (Eq.1) e expressos em \% de lipídios.

Equação 1 - Cálculo para a determinação do total de lipídios excretados (\%).

$$
\% \text { Lipidios totais }=\frac{P L \times 4 \times 100}{P a(g)}
$$

$\mathrm{PL}=$ peso dos lipídios, $\mathrm{Pa}=$ peso da amostra $(\mathrm{g})$

Equação 2 - Cálculo da digestibilidade lipídica

$$
\% \text { Digestibilidade lipídica }=\frac{\text { (lipídios ingeridos }- \text { lipídios excretado }) \times 100}{\text { lipidios ingeridos }}
$$

\subsubsection{Avaliações histopatológicas}

Foram retirados fragmentos do lóbulo direito do fígado de cada animal para realização da avaliação histopatológica. As peças retiradas foram lavadas em solução salina $(0,9 \%)$ e fixadas em solução de formol tamponado (10\%). Em seguida, as peças foram submetidas a um processo de desidratação em série crescente de álcoois e diafanização em xilol, em tempo previamente padronizado para todos os grupos, e então incluídas em parafina. Após inclusão, foram seccionados com $5 \mu \mathrm{m}$ de espessura e corados pelo método da Hematoxilina-Eosina para o exame histológico em microscópio de luz. Os procedimentos foram realizados pela equipe do Laboratório Regional de Diagnóstico da Faculdade de Medicina Veterinária da UFPel, sendo as amostras avaliadas sem o conhecimento prévio dos grupos aos quais pertenciam os animais.

Através das observações histopatológicas buscou-se avaliar: a) integridade ou lesão dos diferentes órgãos; b) presença de qualquer alteração, caracterizando o processo, a distribuição e a extensão; c) presença de degeneração gordurosa hepática (esteatose).

\subsubsection{Determinação de lipídios no fígado}

Foram pesadas amostras de $3 \mathrm{~g}$ dos fígados, originadas da mistura de todos os fígados dos animais do mesmo grupo. A determinação foi realizada, em triplicata, através do método de Bligh \& Dyer (1959) adaptado por Araújo (2009). Os resultados foram expressos em \% lipídios.

\subsection{Análise estatística}

Os resultados foram analisados pelo programa Statistica, versão 7.0 (STATISTICA, 2004). Os dados foram submetidos à análise de variância (ANOVA) através do teste $\mathrm{F}$, a $5 \%$ de probabilidade. A comparação entre os grupos foi feita utilizando-se teste de Tukey $(\mathrm{p}<0,05)$. 


\section{Resultados e discussão}

\subsection{Resultados}

\subsubsection{Avaliações bioquímicas}

Nas Tabelas 2 e 3 estão apresentados os resultados das avaliações séricas, de consumo de dieta, análise nas fezes, no fígado e digestibilidade lipídica das ratas alimentadas com as dietas propostas.

Tabela 2. Avaliações bioquímicas em ratas fêmeas wistar submetidas a diferentes dietas experimentais durante 15 dias.

\begin{tabular}{|c|c|c|c|c|}
\hline \multirow{2}{*}{$\begin{array}{l}* * * \text { Avaliações } \\
\text { Bioquímicas } \\
\left(\mathrm{mg} / \mathrm{dL}^{-1}\right)\end{array}$} & \multicolumn{4}{|c|}{ Dietas } \\
\hline & $\mathbf{C}$ & $\mathbf{A}$ & $* \mathrm{Sm}$ & $* * \mathbf{S n}$ \\
\hline Colesterol total & $77.66 \pm 10.87^{\mathrm{a}}$ & $38.33 \pm 33.92^{b}$ & $93.16 \pm 10.10^{\mathrm{a}}$ & $83.5 \pm 13.21^{\mathrm{a}}$ \\
\hline HDL & $31.66 \pm 4.54^{\mathrm{a}}$ & $28.00 \pm 15.45^{b}$ & $32.66 \pm 4.08^{\mathrm{a}}$ & $31.83 \pm 3.71^{\mathrm{a}}$ \\
\hline LDL & $33.16 \pm 2.64^{\mathrm{a}}$ & $13.66 \pm 9.01^{b}$ & $44.5 \pm 8.87^{\mathrm{a}}$ & $37.00 \pm 13.84^{\mathrm{a}}$ \\
\hline VLDL & $14.5 \pm 4.08^{b}$ & $19.66 \pm 11.49^{\mathrm{a}}$ & $16.00 \pm 5.05^{b}$ & $14.33 \pm 4.68^{b}$ \\
\hline $\begin{array}{l}\text { Triacilgliceróis } \\
\text { (TG) }\end{array}$ & $73.00 \pm 20.66^{\mathrm{a}}$ & $99.00 \pm 58.11^{\mathrm{a}}$ & $80.66 \pm 25.35^{a}$ & $70.00 \pm 22.29^{a}$ \\
\hline Glicose & $102.33 \pm 15.73^{\mathrm{a}}$ & $100.5 \pm 30.61^{\mathrm{a}}$ & $96.57 \pm 12.63^{a}$ & $105.57 \pm 18.43^{\mathrm{a}}$ \\
\hline
\end{tabular}

Média ( $\mathrm{n}=6) \pm$ estimativa de desvio padrão. Letras diferentes na mesma linha indicam diferença significativa pelo teste de Tukey ( $<<0,05)$. C = Caseína; $\mathrm{A}=$ Aprotéica $* * \mathrm{Sn}=$ Spirulina nanometrica e ${ }^{*} \mathrm{Sm}=$ Spirulina micrométrica. $* * *$ Concentrações $(\mathrm{mg} / \mathrm{dL}$ de soro) de colesterol total, LDL - colesterol, HDL - colesterol, VLDL - colesterol, triacilgliceróis e glicose (mg/dL de sangue).

Fonte: Autores.

Tabela 3. Consumo de dieta, fezes excretadas, lipídios ingeridos, excretados e no fígado e digestibilidade lipídica em ratas fêmeas wistar submetidas a diferentes dietas experimentais durante 15 dias.

\begin{tabular}{lcccc}
\hline \multicolumn{1}{c}{ Avaliações } & \multicolumn{3}{c}{ Dietas } \\
\hline Consumo da dieta (g/rato /dia) & $13.89 \pm 0.69^{\mathrm{a}}$ & $6.96 \pm 1.62^{\mathrm{b}}$ & $14.04 \pm 0.78^{\mathrm{a}}$ & $15,63 \pm 1,58^{\mathrm{a}}$ \\
Lipidios da dieta (g/\%) & $5.60 \pm 1.16^{\mathrm{a}}$ & $5.33 \pm 1.04^{\mathrm{a}}$ & $5.63 \pm 0.04^{\mathrm{a}}$ & $5.58 \pm 1.09^{\mathrm{a}}$ \\
Ingesta de lipídios (g/rato/dia) & $0.77 \pm 0.19^{\mathrm{a}}$ & $0.37 \pm 0.11^{\mathrm{b}}$ & $0.78 \pm 0.27^{\mathrm{a}}$ & $0.80 \pm 0.30^{\mathrm{a}}$ \\
Lipídios totais das fezes (g/\%) & $13.02 \pm 0.73^{\mathrm{a}}$ & $13.13 \pm 0.85^{\mathrm{a}}$ & $11.56 \pm 0.57^{\mathrm{b}}$ & $8.94 \pm 0.96^{\mathrm{c}}$ \\
Fezes excretadas (g/rato /dia) & $1.01 \pm 0.03^{\mathrm{c}}$ & $0.52 \pm 0.01^{\mathrm{d}}$ & $2.53 \pm 0,04^{\mathrm{a}}$ & $1.35 \pm 0.04^{\mathrm{b}}$ \\
Lipidio excretado nas fezes (g/rato/dia) & $0.13 \pm 0.03^{\mathrm{b}}$ & $0.07 \pm 0.01^{\mathrm{c}}$ & $0.29 \pm 0.05^{\mathrm{a}}$ & $0.11 \pm 0.03^{\mathrm{b}}$ \\
Digestibilidade lipidica* (\%) & $83.1 \pm 1.25^{\mathrm{b}}$ & $81.1 \pm 3.19^{\mathrm{b}}$ & $62.8 \pm 4.16^{\mathrm{c}}$ & $87.0 \pm 1.10^{\mathrm{a}}$ \\
Lipídios no fígado (g/\%) & $2.95 \pm 0.16^{\mathrm{b}}$ & $8.34 \pm 0.61^{\mathrm{a}}$ & $3.62 \pm 0,39^{\mathrm{b}}$ & $3.73 \pm 0.62^{\mathrm{b}}$ \\
\hline
\end{tabular}

Média (n=6) \pm estimativa de desvio padrão. *(\%) Digestibilidade lipídica $=($ lipídios ingeridos - lipídios excretados) x $100 /$ lipídios ingeridos. $\mathrm{C}=$ Caseína; $\mathrm{A}=$ Aprotéica $; \mathrm{Sm}=$ Spirulina micrométrica, $* * \mathrm{Sn}=$ Spirulina nanométrica.

Fonte: Autores.

Na Figura 1 estão apresentados os níveis médios das determinações de minerais em mg/dL no soro das ratas alimentadas com diferentes dietas experimentais durante 15 dias. 
Figura 1. Níveis médios das determinações de minerais $(\mathrm{mg} / \mathrm{dL}$ no soro) das ratas alimentadas com diferentes dietas experimentais durante 15 dias (média rata/dieta)

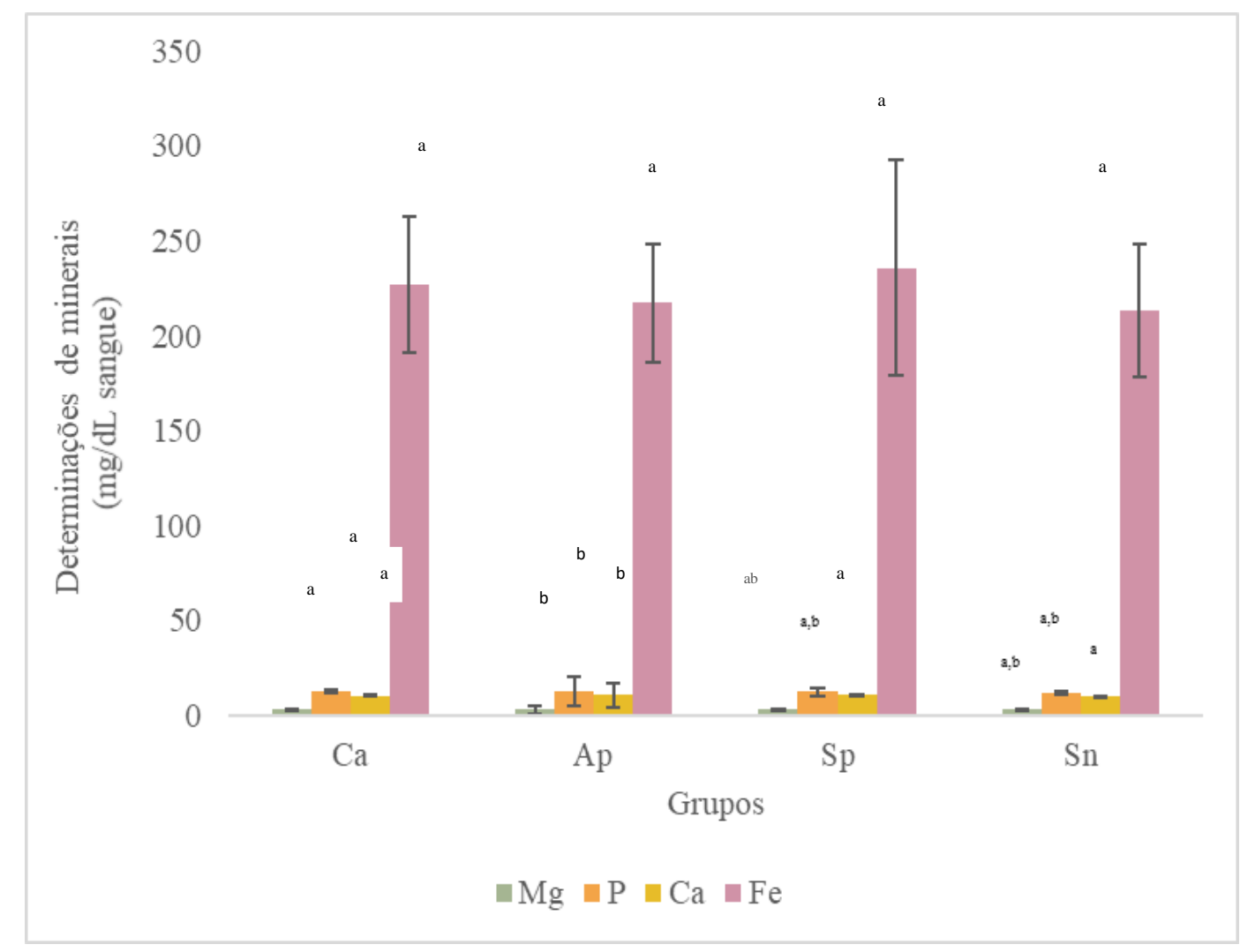

Fonte: Autores.

\subsubsection{Avaliação do perfil histopatológico}

As Figuras 2, 3, 4 e 5 a seguir correspondem às fotos e fotomicrografias de fígados das ratas fêmeas alimentdas com as diferentes dietas em estudo do grupo, Spirulina micrométrica, controle (caseína), Spirulina nanométric e aprotéica. 
Figura 2. Fígado de ratos fêmeas Wistar alimentadas com dieta controle (caseína) por 15 dias. (A) Foto do lóbulo direito do fígado. (B) Fotomicrografia mostrando degeneração vacuolar hepática difusa moderada. (C) degeneração vacuolar hepática multifocal leve e (D) degeneração vacuolar hepática moderada multifocal a coalescente. Aumento na objetiva de 20x.
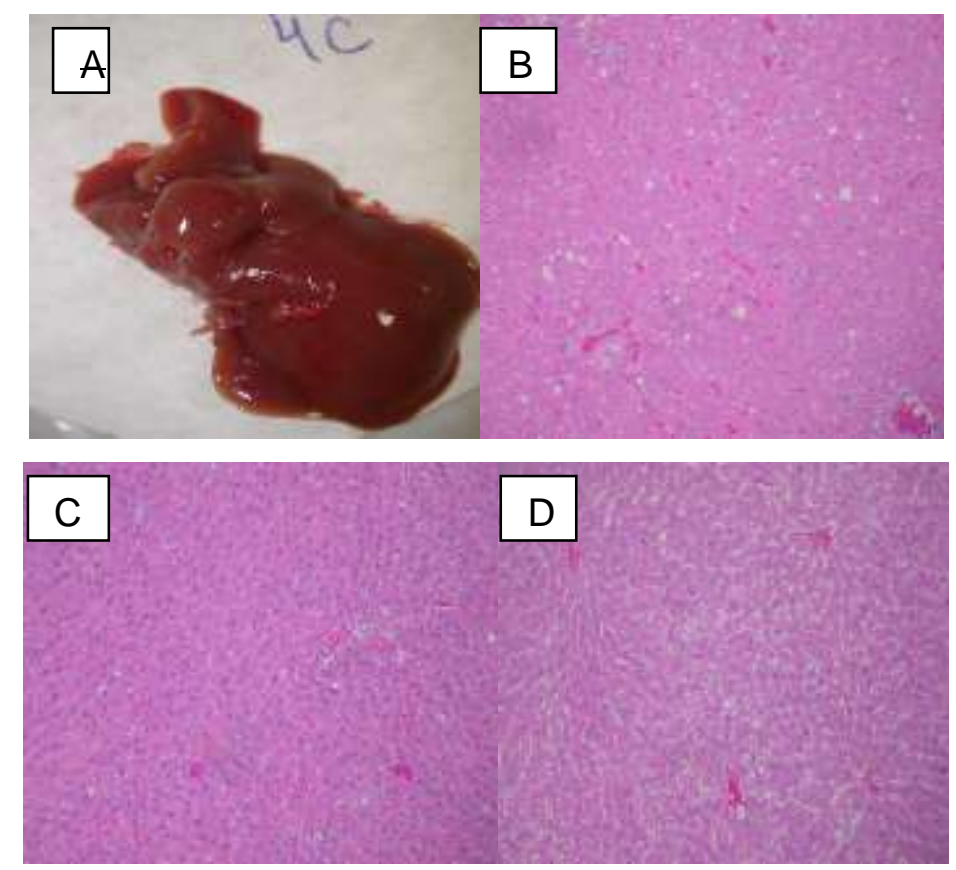

Fonte: Autores.

Figura 3. Figado de ratos fêmeas Wistar alimentadas com dieta aproteica, por 15 dias. (A) Foto do lóbulo direito do fígado. Fotomicrografia (B) e (C) mostrando degeneração vacuolar hepática difusa aguda. (D) degeneração vacuolar hepática coalescente multifocal a aguda de média zona. Aumento na objetiva de 20x.
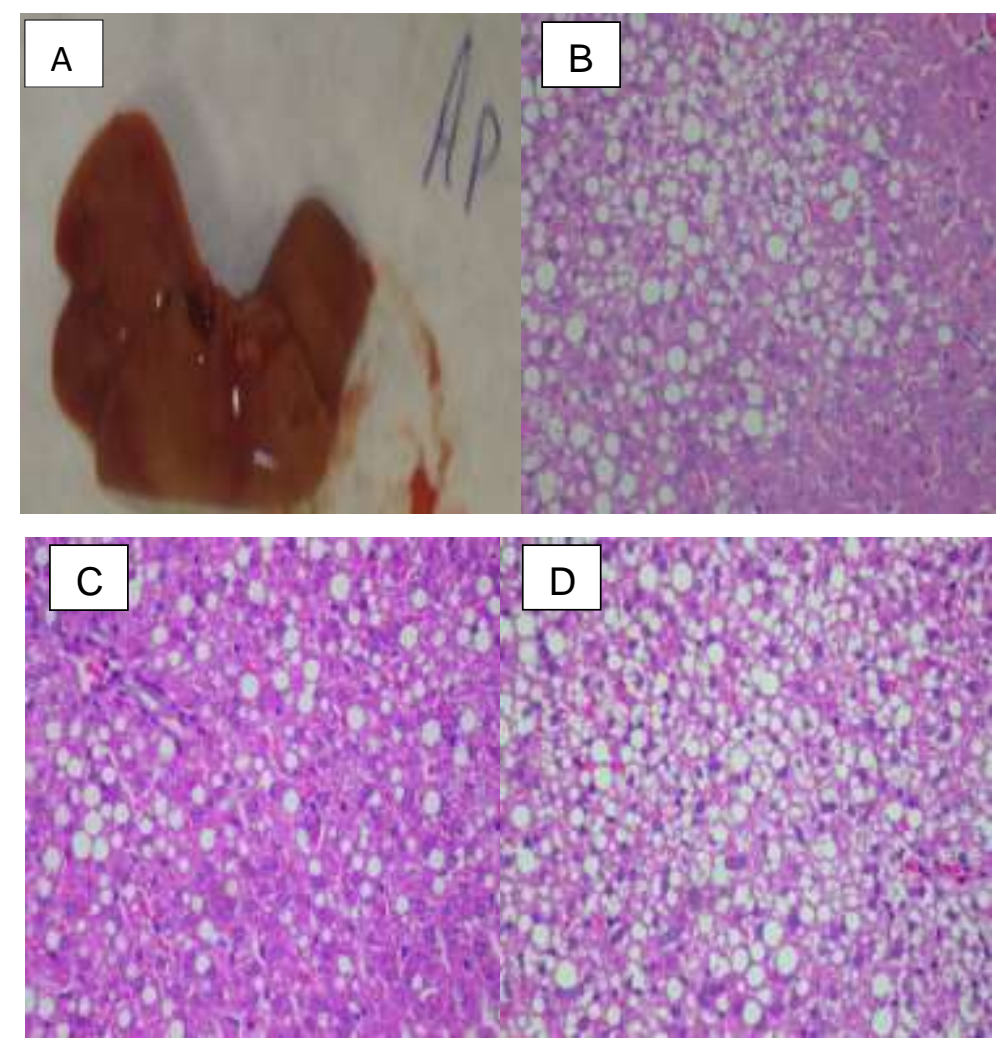

Fonte: Autores. 
Figura 4. Fígado de ratos fêmeas Wistar alimentadas com dieta com Spirulina de tamanho micrométrico, por 15 dias. (A) Foto do lóbulo direito do fígado. (B) Degeneração vacuolar hepática multifocal leve (C) Degeneração vacuolar hepática difusa moderada. (D) Degeneração vacuolar hepática difusa leve. Aumento na objetiva de 20x.
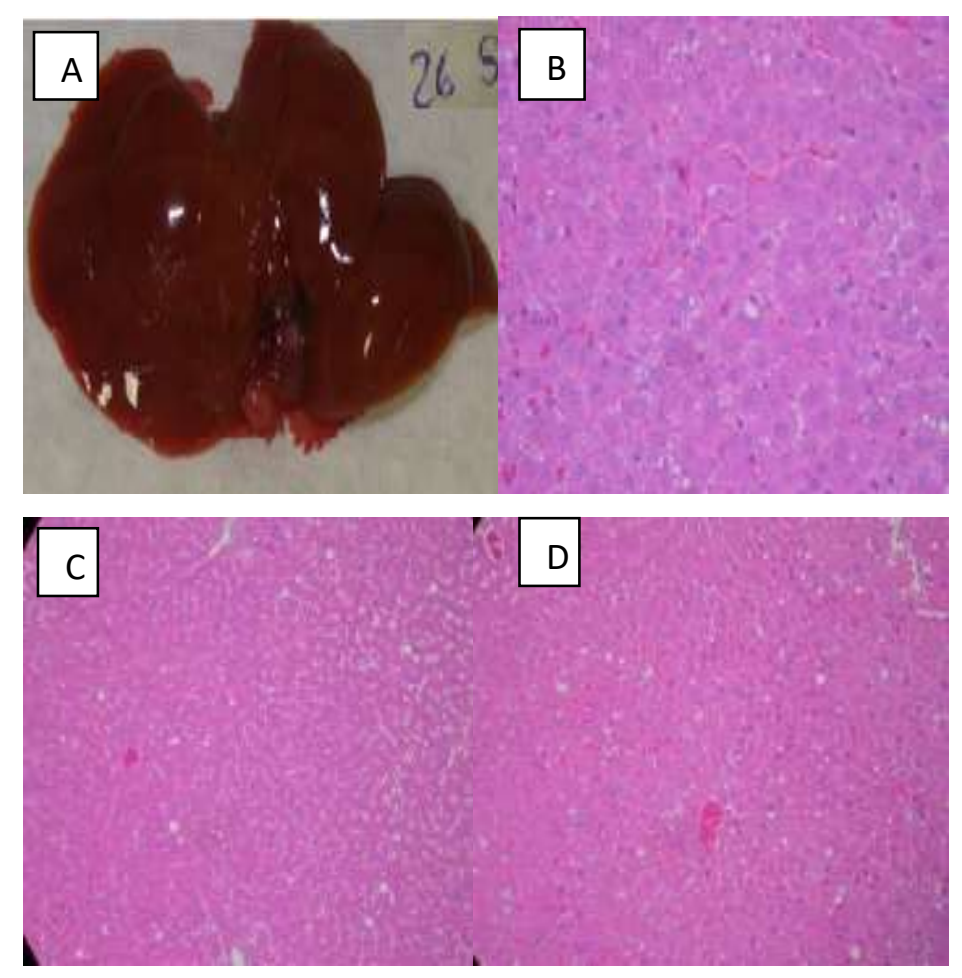

Fonte: Autores.

Figura 5. Fígado de ratos fêmeas Wistar alimentadas com dieta contendo Spirulina nanometrica, por 15 dias. (A) Foto do lóbulo direito do fígado. (B) Fotomicrografia da degeneração vacuolar hepática coalescente multifocal a moderada. (C) Degeneração vacuolar hepática difusa leve. (D) Degeneração vacuolar hepática difusa moderada. Aumento na objetiva de 20x.
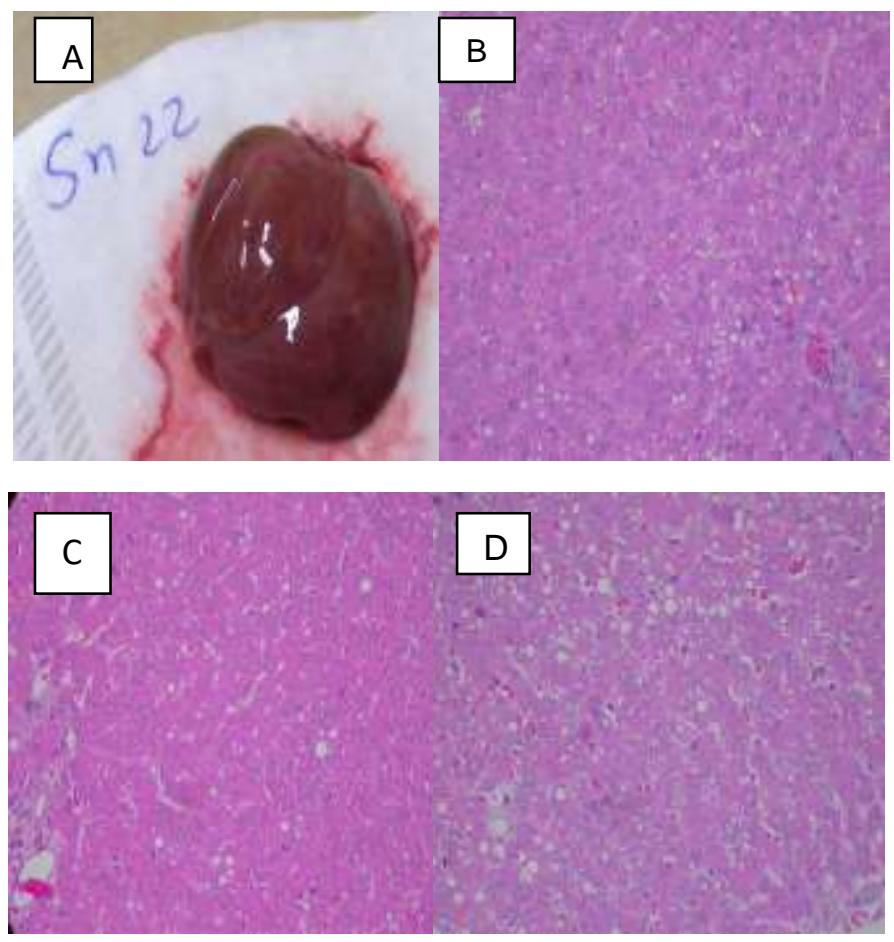

Fonte: Autores. 


\subsection{Discussões}

\subsubsection{Avaliações Bioquímicas}

De acordo com a Tabela 2, os valores séricos do colesterol total e das frações dos ratos com as dietas caseína (C), Spirulina micrométrica (Sm) e Spirulina nanométrica ( $\mathrm{Sn}$ ) não diferiram entre si, apenas o grupo aproteico diferiu dos demais, apresentando desvios padrão muito elevados. A hiperlipidemia caracteriza-se por uma série de distúrbios proporcionados pelo excesso de substâncias, dentre estas o colesterol, triacilgliceróis e lipoproteínas do plasma sanguíneo, como HDL (lipoproteína de alta densidade), LDL (lipoproteína de baixa densidade) e VLDL (lipoproteína de densidade muito baixa), sendo um relevante fator de risco no desenvolvimento de doenças cardíacas e de arteriosclerose (Chiattone et al., 2015).

Embora a microalga Spirulina contenha compostos antioxidantes como a ficocianina e compostos fenólicos que estão ligados à diminuição do colesterol no sangue, essa ação não foi verificada no estudo. O pouco tempo de submissão dos animais às dietas e o fato de que as respostas mais conclusivas surgem nas dietas hipercolesterolêmicas explicam esse comportamento. Essas declarações são mais evidentes ao analisar os resultados de Cheong et al. (2010), cujos coelhos foram alimentados com uma dieta rica em colesterol, mostrando que a suplementação com Spirulina reduziu os níveis de triacilgliceróis e LDLcolesterol no sangue, e Colla et al. (2008), em que coelhos alimentados com dietas ricas em colesterol mostraram uma diminuição em seus níveis de lipoproteínas no sangue quando alimentados com dietas adicionadas com 0,5 g / dia de Spirulina. No entanto, Bertolin et al. (2009) estudaram o efeito de Spirulina platensis no perfil lipídico de ratos Wistar e não observaram alterações significativas quando os trataram com dieta hipercolesterolêmica e Spirulina, por 60 dias, indicando que as microalgas associadas a uma dieta rica em colesterol não alteraram os níveis lipídicos.

De acordo com a literatura, um aumento nos níveis de HDL está associado a uma maior proteção contra a aterosclerose, ou seja, na patogênese da aterosclerose, a lipoproteína de alta densidade (HDL-c) apresenta um importante papel protetor, pois transporta o excesso de colesterol das artérias e da corrente sanguínea até o fígado, onde é metabolizado e eliminado sob a forma de ácidos e sais biliares (Duarte et al.,2009).

Enquanto um aumento na LDL está associado ao aumento dos riscos de aterosclerose, da mesma forma, níveis altos de colesterol e de soro VLDL indicam um aumento risco aterogênico (Grundy, et al. 2014; Batista et.al. 2018).

Na Tabela 2, observa-se que dietas com Spirulina em diferentes tamanhos resultaram em níveis de HDL semelhantes ( $p<0,05)$ ao controle. Chiattoni et al., (2015) encontraram níveis de HDL igual a 30,5 mg.dL-1, nos animais do grupo controle, e em seus tratamentos 28,$15 ; 34,16$ e 23,65 mg.dL-1, nos animais alimentados com dietas acrescidas de diferentes concentrações de Spirulina.

A dieta aproteica aumentou as concentrações de TG e VLDL e as dietas Controle, Sm e Sn resultaram em maiores concentrações de LDL (Tabela 2), que podem ser associadas com a preferência alimentar demonstrada pelos grupos experimentais (Di Nicolantonio et al., 2016).

Animais de diferentes grupos não diferiram estatisticamente em relação aos triacilgliceróis, estando os valores de acordo com as recomendações do Centro de Bioterismo da USP (FMUSP, 2011) para Rattus norvegicus saudáveis de 66,35 a $78,00 \mathrm{mg} \mathrm{dL}^{-1}$. Da mesma forma, os grupos experimentais não diferiram estatisticamente (p>0,05) em relação à concentração de glicose no sangue. As maiores concentrações, numericamente, foram observadas nos grupos submetidos às dietas (C) e $(\mathrm{Sn})$, e a menor média foi demonstrada no grupo que recebeu dieta $(\mathrm{Sm})$. Provavelmente, isso se deve à influência dos outros ingredientes da composição da dieta (Tabela 1). Além disso, um maior teor de fibra (particularmente fibra solúvel) promove uma camada de gel protetor no intestino, oferecendo assim um grande obstáculo à captação de glicose, causando menor índice glicêmico no sangue (Chaud et al., 2008). Geralmente todos os grupos apresentaram níveis normais de glicose no sangue para a espécie, $50 \mathrm{mg} \mathrm{dL}^{-1}$ a $135 \mathrm{mg} \mathrm{dL}^{-1}$ (Harkness e Wagner, 1993). Outros autores, como Aires-Neto (2005), encontraram glicose 
variando de 96 a $102 \mathrm{mg} \mathrm{dL}^{-1}$ em ratos machos e Nogueira -Junior et al. (2005), trabalhando com ratos fêmeas, encontraram glicemia menor que $130 \mathrm{mg} \mathrm{dL}^{-1}$.

De acordo com a Tabela 3, o consumo de lipídios na dieta foi o mesmo para todos os grupos. A recuperação lipídica, ou digestibilidade lipidica, dos animais foi significativamente maior com a dieta baseada em Spirulina do tamanho nanômetrico. Provavelmente, isso se deve à suplementação com caseína na formulação dessa dieta, assemelhando-se às dietas controle (Tab.3).

O grupo que consumiu Spirulina do tamanho de micrômetros obteve um aumento no volume de fezes excretadas e principalmente no conteúdo de lipídios excretados pelas mesmas (Tab.3).

A digestibilidade lipídica depende do pleno funcionamento de vários órgãos do trato gastrointestinal, e quaisquer alterações relacionadas a ele podem afetar a digestão total, absorção e metabolismo dos nutrientes (D'Agostin, 2001; Kierzabaum, 2004).

Pode-se observar que as dietas controle, aproteica e nanométrica de Spirulina apresentaram percentual aproximado de digestibilidade lipídica, enquanto que a dieta contendo Spirulina do tamanho de micrômetros apresentou uma porcentagem menor, provavelmente devido ao teor de fibras presentes.

A maior concentração de lipídios hepáticos foi encontrada nos animais que receberam dieta aproteica. Os animais cujas dietas continham Spirulina apresentaram menor quantidade de lipídios hepáticos, característica das dietas de origem vegetal, indicando que as dietas deste estudo $(\mathrm{Sm}, \mathrm{Sn})$ apresentaram um efeito benéfico devido à menor deposição lipídica no fígado. O mesmo ocorreu no trabalho de Torres - Duran et al. (2007) onde a Spirulina maxima fornecida por via oral proporcionou um efeito hepatoprotetor na manutenção do perfil lipídico do fígado.

\subsubsection{Níveis séricos de minerais}

Um grande número de minerais é reconhecido como essenciais para manter a vida. Pode-se classificá-los em 2 grandes grupos, sendo a quantidade encontrada no organismo o marco divisor. Os oligoelementos são encontrados em quantidade menores que $100 \mathrm{mg} / \mathrm{kg}$ de peso corpóreo total ( $\mathrm{Fe}, \mathrm{Zn}, \mathrm{Cu}, \mathrm{Mn}, \mathrm{Cr}$, Mo, Se, F, I, etc). Na avaliação do estado nutricional dos minerais, vários fatores são considerados, como, por exemplo, edema e função renal. Para alguns, o Mg e o P diminuem a sua excreção urinária com a menor oferta. Neste estudo os grupos contendo spirulina não diferiram estatisticamente dos grupos caseina e aprotéica, porém estes grupos apresentaram diferença entre eles com relação aos minerais séricos $\mathrm{Mg}, \mathrm{P}$ e $\mathrm{Fe}$ e $\mathrm{Ca}$. Sendo que o teor de cálcio encontrado no plasma sanguíneo, apenas houve diferença no grupo aproteico. Conforme Cisternas, Vargas e Monte (2001), o nível de cálcio em sangue humano varia de 9 -11 mg/dL. Feddern (2007) obteve valores entre 9,8 e 10,9 mg. $\mathrm{dL}^{-1}$ para ratas Wistar alimentadas com dietas de diferentes misturas. Santos et al.(2004), em sua pesquisa sobre o estudos bioquímicos e hematológicos em ratos sobre biodisponibilidade de minerais numa dieta enriquecida com multimistura, verificou os niveis de minerais no soro para grupos que receberam dietas creche com multimistura e apresentaram o teor de cálcio (Ca) e magnésio ( $\mathrm{Mg}$ ) respectivamente 10,09 e 3,04, estando proximos aos deste estudo (Mg para caseína, aproteica, spirulina micrométrica e spirulina nanometrica, respectivamente 3,29; 3,28; 3,16 e 3,11 e Ca para caseina, aproteica, spirulina micrométrica e spirulina nanometrica , respectivamente, 10,71; 10,75; 10,33 e 10,70).

\subsubsection{Avaliação histopatológica do fígado}

Os exames histopatológicos de órgãos, como fígado, permitem uma fácil detecção de anormalidades em caso de toxicidade (Bisar et al. 2017). Nesta pesquisa, a observação microscópica de tecido hepático de ratos controlados e tratados com com as diferentes dietas em estudo como: controle (caseína), aprotéica, Spirulina micrométrica e Spirulina nanométrica. 
Nas figuras (2,3,4 e 5) do lóbulo direito do fígado, pode-se observar que praticamente todos os grupos apresentaram algum grau de lesão hepática.

Para ratos que receberam dietas à base de caseína, o fígado apresentou $50 \%$ de lesões leves a moderadas (Fig. 2). Os animais que receberam dieta aproteica (Fig. 3) apresentaram lesões hepáticas muito marcadas (100\%). As lesões hepáticas foram muito heterogêneas em relação ao padrão e intensidade. Isso pode ser devido à redução / restrição da ingestão de proteínas. No entanto, nos fígados de ratos que receberam dieta com Spirulina nos tamanhos micrométrico (Fig. 4) e nanométrico (Fig. 5), as lesões correspondem a 20\% (leve) a 80\% (moderada).

Nos fígados não foram observadas lesões patológicas significativas. Uma das alterações metabólicas nas células é o acúmulo intracelular de substâncias, como água, lipídios, carboidratos e proteínas. O significado dessa acumulação (degeneração) depende da causa e gravidade da deposição. Quando é suave, pode não causar efeito na função celular. Um acúmulo intracelular acentuado (grave) pode prejudicar a função levando à morte celular. É importante ressaltar que na hematoxilina e eosina não é possível determinar qual substância está se acumulando (Schild, 2011).

Ferreira - Hermosillo et al. (2010) estudaram pacientes com doença hepática gordurosa não alcoólica, e que receberam 4,5 g de Spirulina diariamente, por três meses, concluíram que Spirulina pode ser considerada como um tratamento alternativo para pacientes que sofrem de doença hepática gordurosa não alcoólica ou distúrbios de dislipidemia. Chen et al. (2019) concluíram que a suplementação de extratos etanólicos de Spirulina na dieta de ratos Wistar machos durante oito semanas diminuiu a ocorrência de lesões no fígado e melhorou a anormalidade dos hepatócitos. Guzmán-Gómez et al. (2018) avaliaram a atividade das enzimas de defesa antioxidante, o nível de peroxidação lipídica e as alterações histopatológicas da mucosa gástrica de ratos Wistar machos após a administração intragástrica de ficobiliproteínas extraídas de Spirulina Maxima na dosagem de 100, 200 ou $400 \mathrm{mg} / \mathrm{kg}$ de corpo peso. Os autores relataram valores de índices reduzidos a respostas inflamatórias, especialmente após a administração na concentração de $400 \mathrm{mg} / \mathrm{kg}$ de peso corporal.

A esteatose hepática ou acúmulo de gordura no fígado é um distúrbio metabólico que também foi observado em organismos submetidos à desnutrição crônica de proteína - energia. Esse aumento da concentração de gordura no fígado ocorre principalmente devido ao aumento de triacilgliceróis (Kumar 1972, Taylor e Ziboh 1972). Efetivamente, o grupo aproteico mostrou expressão aumentada de lesão hepática (Fig. 2) acompanhada de alta concentração de lipídios nesse órgão (Tabela 3) e, embora não significativo, maior concentração de triacilgliceróis no sangue (Tabela 2)

As principais causas da esteatose são a hipoxemia (a gordura transportada para o fígado não é oxidada e é retida); tóxicos (arsênico, clorofórmio, tetracloreto de carbono, micotoxinas) e fatores lipotrópicos insuficientes (colina e metionina) (Coelho et al., 2002).

\section{Conclusão}

As dietas contendo Spirulina cepa LEB-18 nos tamanhos micrométrico e manométrico não alteraram os parâmetros bioquímicos (colesterol total e frações, triacilgliceróis e glicose) de ratos Wistar fêmeas em crescimento. No entanto, verificouse que os animais apresentaram algum grau de lesão hepática, embora bastante heterogêneos quanto ao padrão e intensidade. Isso pode ser devido à deposição lipídica registrada na dieta contendo Spirulina de tamanho nanométrico, sendo mais evidente na dieta aproteica. Por fim, nossos resultados fornecem subsídios para estudos futuros, mais detalhados, incluindo técnicas sofisticadas como a cromatografia gasosa para o perfil em ácidos graxos de cada dieta com fonte lipídica, assim como determinar, pela mesma tecnica, perfil de ácidos graxos teciduais, como o fígado. 


\section{Agradecimentos}

Os autores agradecem a CAPES, CNPQ, e FAPERGS pelo apoio financeiro.

\section{Conflitos de interesse}

Os autores declaram não haver qualquer potencial conflito de interesses que possa Interferir na imparcialidade deste trabalho Científico.

\section{Referências}

Aires-Neto,T. A., Cavalcante, J. F., Brandão-Neto, J., Araújo-Filho, I., Almeida, M. D. A. S. G.,Rezende, A. A., et al. (2005). Total gastrectomy with substitution of stomach by jejunal pouch with and without duodenal passage.study in rats. Acta Cirurgica Brasileira.; 20,supl.1. 107-112.

Araújo, P. F. (2009). Atividade antioxidante de néctar de amora-preta (Rubus spp.) e sua influência sobre os lipídios séricos, glicose sanguínea e peroxidação lipídica em hamsters (Mesocricetus auratus) hipercolesterolêmicos.123f. Dissertação (Mestrado) - Programa de pós-graduação em Ciências e Tecnologia Agroindustrial. Universidade Federal de Pelotas, Pelot

Ax, E., Cederholm, T., Grundmark, B., Bill-Axelson, A., \& Becker, W. ( 2013).Dietary Patterns and prostate cancer risk: a population based cohort study in elderly Swedish men. The FASEB Journal. 27(S1): 847.8.

Batista, K. S., Alves, A. F., dos Santos Lima, M., da Silva, L. A., Lins, P. P., de Sousa Gomes, J. A., . \& da Conceição, M. L. (2018). Beneficial effects of consumption of acerola, cashew or guava processing by-products on intestinal health and lipid metabolism in dyslipidaemic female Wistar rats. The British journal of nutrition, $119(1), 30$.

Belay, A.(1997). Mass culture of spirulina in outdoors - The earthrise farms experience. In: Vonshak, A. (ED.). Spirulina platensis (arthrospira): physiology, Cellbiology and biotechnology. London, Ed.Taylor And Francis. 131-158.

Bertolin, T. E., Pilatt, I. D., Giacomini, A. C. V. V., Bavaresco, C. S., Colla, L. M., \& Costa, J. A. V. (2009). Effect of microalga Spirulina platensis (Arthrospira platensis) on hippocampus lipoperoxidation and lipid profile in rats with induced hypercholesterolemia.Braz.Arch.Biol.Technol.;53(5):1253-1259.

Bisar, G. H., Youssef, M., Saadany, K. E., El-Kholy, W., Kheadr, E. (2017). Effect of lentil and buffalo protein hydrolysates on histopathology of liver and kidney in diabetic rats. Journal Cytol. Histol., 8:489.

Bligh, E. G. Dyer, S. W. J. (1959). A rapid method of total lipid extraction and purification. Canadian Journal of Biochemistry and Physiology. 37(8), 911917.

Chaud, S. G., Sgarbieri, V. C., \& Vicente, E. (2008). Influência de frações da parede celular da levedura (Saccharomyces,cerevisae) sobre alguns parâmetros nutricionais de ratos em crescimento. Revista de Nutrição. 21(2):137-147.

Chen, H., Zeng, F., Li, S., Liu, Y., Gong, S., Lv, X., Zhang, J., \& Liu, B. (2019). Spirulina active substance mediated gut microbes improve lipid metabolism in high-fat diet fed rats. Journal of Functional Foods. 59:215-222.

Cheong, S. (2010).Spirulina Prevents Atherosclerose By Reducing Hypercholesterolemia In Rabbits Fed A Hight -Cholesterol Diet, Journal of Nuitrition Science and Vitamology. 56(10):34-40.

Chiattoni, L. M., Machado, M. R. G., Rodrigues, R. D. S., \& Soares, L. A. D. S. (2015). Influência do consumo de diferentes níveis de Spirulina no desenvolvimento e perfil lipídico de ratos. Revista Ceres, 62(2), 142-148.

Coelho, H. E.(20002). Patologia Veterinária: Manole. 234p.

Colla, L. M., Muccillo-Baisch, A. L., \& Costa, J. V.(2008). Spirulina platensis effects on the levels of total cholesterol, HDL and triacylglycerols in rabbits fed with a hypercholesterolemic diet. Braz.Arch Biol Technol.51(2):405-11.

Costa, J. A. V., Colla, L. M., Duarte-Filho, P. F. (2004). Improving Spirulina platensis biomass yield using a fed-batch process. Bioresource Technology, Amsterdan .(92):237-241.

Cushen, M., Kerry, J., Morris, M., Cruz-Romero, M., \& Cummisns, E. (2012). Nanotechnologies in the food industry a recent developments, risks and regulation. Trends in Food Science \& Technology. 2012; (24): 30 - 46.

Duarte, S. M. S., Abreu, C. M. P., Menezes, H. C., Paula, F. B. A., Pereira, R. G. F. A \& Gouvea, C. M. C. P. (2009). Peeled coffee brew effect in the antioxidant activity hematological and biochemical parameters in rats. Ciência e Tecnologia de Alimentos, 29:703-708.

DiNicolantonio, J. J., Lucan, S. C., \& O'Keefe, J. H. (2016). The evidence for saturated fat and for sugar related to coronary heart disease. Progress in cardiovascular diseases, 58(5), 464-472.

Ferreira-Hermonillo, A., Torres-Duran, P., \& Juarez - Oropeza, M.( 2010). Hepatoprotective Effects of Spirulina máxima In Patients With Non Alcoholic Fatty Liver Disease:A Case Series, Journal of Medical Case Reports.7:4:103.

FMUSP - Centro de Bioterismo da Faculdade de Medicina da Universidade de São Paulo. http://www.biot.fm.usp.br/site1/index-2.html. 
Research, Society and Development, v. 10, n. 1, e50310111654, 2021

(CC BY 4.0) | ISSN 2525-3409 | DOI: http://dx.doi.org/10.33448/rsd-v10i1.11654

Guzmán-Gómez, O., García-Rodríguez, R. V., Quevedo-Corona, L., Pérez-Pastén-Borja, R., Rivero-Ramírez, N. L., Ríos-Castro, E., Pérez-Gutiérrez, S., Pérez-Ramos, J., \& Chamorro-Cevallos, G. A. (2018). Amelioration of Ethanol-Induced Gastric Ulcers in Rats Pretreated with Phycobiliproteins of Arthrospira (Spirulina) Maxima. Nutrients.10,763.

Grundy, S. M., Arai, H., Barter, P., et al. (2014) An International Atherosclerosis Society Position Paper: global recommendations for the management of dyslipidemia - full report. J Clin Lipidol 8, 29-60.

Harkness, J., Wagner, J. E. (1993); Biologia e clinica de coelhos e roedores.Rocca (3 ${ }^{\mathrm{a}}$ ed.).

Kumar, V., Deo, M. G., \& Ramalingaswami, V. (1972). Mechanism of fatty liver in protein deficience: an experimental study in the rhesus monkey. Gastroenterology. 62:445-51.

Machado, A. R., Assis, L. M., Machado, M. R. G. and Souza-Soares, L. A. (2015). Biological evaluation of casein and Spirulina in rats and these protein sources encapsulation in liposomes. International Food Research Journal. 22(1): 338-344.

Machado, A. R., Graça, C. S., Assis, L. M. D., \& Souza-Soares, L. A. D. (2017). Uma abordagem sobre caracterização e avaliação do potencial antioxidante de extratos fenólicos de microalgas Spirulina sp. LEB-18 e Chlorella pyrenoidosa. Revista de Ciências Agrárias, 40(1), 264-278.

Morais, M. G, Costa, J. A. V. (2008). Perfil de acidos graxos de microalgas cultivadas com dióxido de carbono. Ciência Agrotecnica de Lavras. (32): 12451251.

Nogueira Junior, F. C., Coelho, D. A., Almeida, M. M. C., Silva, T. C. P, Ferreira, E. C. S., Macedo, U. B. O., et al. (2005). Efeito do B tamoxifeno no perfil lipídico dos ratos diabéticos por estreptozotocina. Acta Cirurgica Brasileira. 20,supl.n.1.

Pereira, A. S. et al. (2018). Metodologia da pesquisa científica. [e-book]. Santa Maria. Ed. UAB/NTE/UFSM. https://repositorio.ufsm.br/bitstream/handle/1/15824/Lic_Computacao_Metodologia-Pesquisa-Cientifica.pdf?sequence=1.

Reeves, P. G., Nielsen, F. H., \& Fahey, G. C. (1993). Ain-93 purified diets for laboratory rodents: final report of the American Institute of Nutrition ad hoc writing committee on the reformulation of the AIN-76a rodent diet. J. Nutrition. 123(10):939-1951.

Rodriguez-Hernandez, A., Blé-Castillo, J. L., Juárez-Oropeza, M. A., \& Diaz-Zagoya, J. C. (2001); Spirulina maxima prevents fatty liver formation in CD-1 male and female mice with experimental diabetes. Life Sciences. 69:1029-37.

Schild, A. L., e Colaboradores. (2011). Laboratório Regional de Diagnóstico, comunicação pessoal.

Statistica,Statsoft (dataanalysis software system)versin 7 for Windows.www.statsoft.com 2004.

Taylor, G. O., Ziboh,V. A.( 1972). Liver lipid changes in experimental protein malnutrition. Am. J. Clin.Nutr.25:285-90.

Torres-Duran, P. V., Ferreira-Hermosillo, A., \& Juarez-Oropeza, M. A. (2007). Antihyperlipemic and antihypertensive effects of Spirulina maxima in an open sample of Mexican population: a preliminary report. Lipids Health Dis.; 6:33. 